\title{
Salt contamination calculation in insulators during monsoon using artificial neural network
}

\author{
Jewan Singh*, Vibhakar Mansotra \\ Information Technology, AMET University, Chennai. \\ Department of computer science \& IT, University of Jammu, Kashmir
}

\begin{abstract}
Control framework consistent quality depends mostly on the natural and climate conditions which cause flashover on contaminated protectors. Such flashover prompts framework blackouts. Close to the beach front zones the salt pollution can be quickly based on the surface of the shields and frame a directing layer by retaining wet from the fog. This layer at times prompts flashover. Investigation of defilement of separator under marine contamination is the point of this examination, and the impacts of different meteorological elements on the disease seriousness have been researched altogether. In the present paper, an endeavour has been made to gauge the contamination severity under different climate conditions amid the stormy season utilising Artificial Neural Organize. The anomaly determination issue has been considered in this work to take out few exceedingly scattered exploratory information. The connection between ESDD with temperature $T$, stickiness $H$, weight $P$, precipitation $R$ and wind speed $W V$ has been created utilising ANN as a function estimator
\end{abstract}

Keywords: neural networks, salt contamination

\section{Introduction}

The electrical power framework lines once in a while go along the waterfront locales of the Peninsular Malaysia. Salty winds blew from the South China Sea toward the eastern place that is known for the promontory cause a salt statement on power framework separators [1]. The salt report, in the end, polluting the cover surfaces prompts protection flashover. This layer gives a perfect way for the spillage current to go through between the high voltage side also, the ground side. The conductivity of the tainting layer relies on upon the kind of salts which this layer comprises of High disappointment rate of contaminated separator because of the flashover has been found close to the beachfront territories [2]. Pollution observing is required keeping in mind the end goal to decide the best possible outline and a decision of protection sort and level. This observing is additionally critical for tending to a great arrangement against contamination flashover. Meteorological conditions change impressively from the seaside zones to the inland regions and play a crucial part in the affidavit rate of toxins and electrical conductivity of covers [3]. The study demonstrated that the cover defilement issue is emphatically conditioning subordinate and no summed up hostile to contamination criteria can be advertised [4]. The issue progresses toward becoming more genuine at more elevated amounts of transmission voltage where the higher protection level is not down to earth, in fact, and monetarily [5]. It is, consequently, basic to have a reasonable and exact evaluation of site seriousness. Works in have discovered meaningful connections between the sullying seriousness regarding Equivalent Salt Deposit Density (ESDD) and flashover as for the meteorological parameters [6]. And no more the examination can concentrate the impact of one or, on the other hand, a greater amount of these parameters on ESDD or flashover [7]. Endeavors have been done in to relate the majority of the meteorological parameters with ESDD and build up a new numerical model utilising many relapse investigation technique. The eastern district of the Peninsular Malaysia is considered as a highly contaminated region because of salty wind originating from the South China Sea [8]. In Malaysia, a significant portion of the power era stations is worked close to the beachfront regions. Thus, the high voltage protectors introduced in these areas ought to be considered genuinely regarding different parts. The site estimation of the contamination seriousness regarding ESDD has been completed with average pressure sort Cap-and-Pin glass protectors amid cold season under five differing meteorological components, for example, temperature, moistness, pneumatic force, precipitation and wind speed. In the most recent decade, many research has been done on the 
utilisation of ANN in many fields. It has been utilised effectively in capacitor control, a uniform, what's more, complex electric anxiety circulation along the protector surface [IZ], alert preparing, in example acknowledgement of halfway releases and contamination release demonstrating. In this paper, another approach utilising ANN as capacity estimator has been created and used to show precisely the relationship between ESDD (the reliant variable) and the meteorological parameters (the free elements) for example, temperature, moistness, weight, precipitation, and wind speed. Displaying approach given ANN is utilised as a capacity estimator to demonstrate the pollution level of high voltage separators under different meteorological parameters. Bioprospecting of Halotolerant Marine Bacteria from the Kelambakkam and Marakkanam Salterns [9]. Production and Purification of Biosurfactant from Marine Yeast Isolated from Kelambakkam Salterns [10]. Among the different ANN structures, the multilayer encourages forward system with back-engendering is decided for managed learning. The back-engendering calculation is utilised in the preparation procedure to determine the association weights of the neural system. It is discovered that the ANN demonstrating is hugely successful and precise.

\section{Proposed Methodology}

The approach that has been utilised to address the contamination seriousness at the test area is called Equivalent Sal4 Store Density (ESDD) expressed in units of $\mathrm{mg} / \mathrm{cm} \mathrm{NaCl}$. ESDD is the same measure of $\mathrm{NaCl}$ that would yield a similar conductivity at finish weakening. Comparative Study of Neural Networks Algorithms for Cloud Computing CPU Scheduling is discussed in [11]. The elucidation of ESDD information might be not quite the same as the place to put its esteem yet gives a premise of an order of tainting seriousness. ESDD is broadly utilised as a part of Malaysia for deciding salt tainting level. Foreign Tourist Arrivals Forecasting Using Recurrent Neural Network Backpropagation through Time is illustrated in [12]. If \$he estimation of ESDD is equivalent or more noteworthy than $0.03 \mathrm{mg} / \mathrm{cm}$, the separators are then washed. The site evaluation exercises are completed every day amid dry season at Sultan Ismail Power Station in Paka, Terengganu, Malaysia using three specimens of the run of the mill pressure sort Cap-and-Pin glass separators which are usually introduced on transmission lines in that range. The examples were brought down from the platform, and the toxins were evacuated by washing the encasings utilising paintbrush and pure water. Each debased example for each test was removed by drenching it in distilled water, and the defilement esteem was measured by deciding the conductivity or the rate of ascent of the conductivity esteem for the dirtied water after washing the protector. Utilizing such technique, ESDD can be resolved. The information about defilement conduct and level will help in the foundation of upkeep approach because both first months and introduction times of the year can be gotten. The contamination seriousness is measured as far as ESDD under five fluctuating meteorological elements, e.g. temperature (T), dampness $(H)$, weight $(P)$, precipitation $(R)$ what's more, wind speed (WV). Proficient demonstrating of contamination seriousness and flashover voltage is of central enthusiasm to all architects required in the outline of transmission line separators. Among the different fake neural system introduced up until this point, the multi-layer encourage forward system with back spread strategy is utilised in the present review to demonstrate $\mid 3 s D D=f 6 f l R p \sim W V$ ). The neural system is prepared with the help of information acquired from site estimation, and the preparing exactness has been evaluated by a root mean square mistake (RMSE). Goes about as a model of certifiable framework or capacity. The display then stands for the context it speaks to, ordinarily to foresee or to control it. ANN can show a function regardless of the possibility that the condition depicting it is obscure the main essential is the illustrative example of the capacity conduct, and that is from the test information and not from a hypothetical comprehension. Figure 1 demonstrates the schematic chart of a multi-layer bolsters forward organise utilised as a part of this paper. The neurons in the system can be separated into three layers: the input layer, yield layer, what's more, shrouded layers.

\section{ANN Controller}

Simulated neural system calculation has been utilised effectively in numerous applications. It is valuable since it goes about as a model of certain framework or capacity. The demonstrate then stands for the context it speaks to, usually to anticipate or to control it. ANN can explain a function regardless of the possibility that the condition was portraying it is obscure 
the main essential is the illustrative specimen of the capacity conduct and that is from the exploratory information and not from a hypothetical comprehension. Figure 1 demonstrates the schematic graph of a multi-layer nourishes forward arrange utilised as a part of this paper. The neurons in the system can be isolated from three layers: the input layer, yield layer furthermore, concealed layers. Note that the bolster forward system signs can just spread from the information layer to the yielding layer through the shrouded layers. Every neuron of the yielding layer gets a flag from all information through concealed layer neurons along associations with modifiable weights. The neural system can distinguish input design vectors, once the connection weights are balanced by

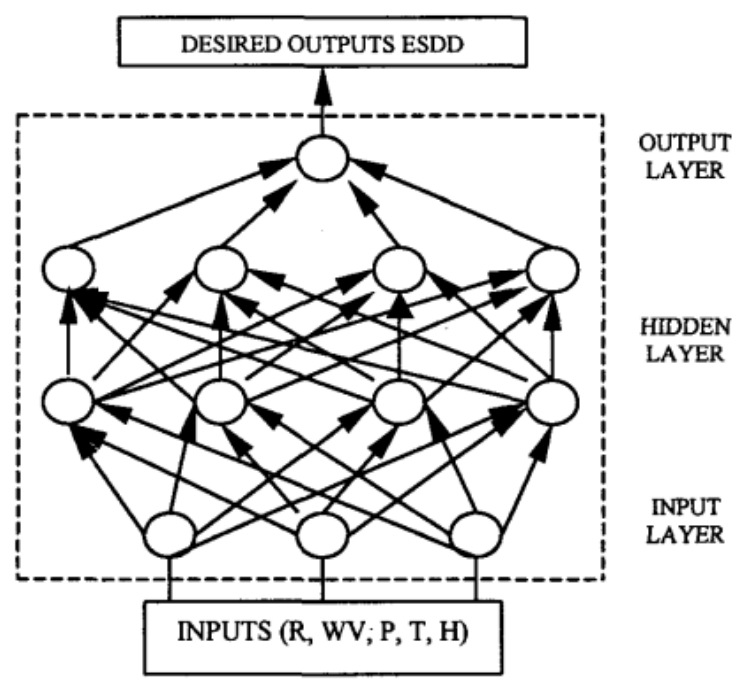

Figure 1. Overall structure of three-layer artificial neural network

Methods for the learning procedure. The back-spread learning calculation which is a speculation of Widrow-Hoff mistake rectification govern is the most mainstream strategy in preparing the ANN and is utilised in this work. This learning calculation is exhibited here in brief. Know that the edge of every neuron is learned in the route same as that for other weights. The side of a neuron is viewed as a modifiable association weight between that neuron and an imaginary neuron in the past layer, which dependably has a yield estimation of solidarity. The preparation exactness of the ANN is measured with the assistance of RMSE (Root Mean Square Mistake). Once the system is prepared, it is tried utilising test information designs and the viability is judged on the premise of \%MAE (Mean Absolute Error).

\section{Results and Discussion}

In applying the learning standard portrayed, there are a few issues which ought to be tended to. The improvement handle has been completed given \%MAE and legs swaying in the blunder of merging. From the outcomes in Tables 1-2, the perceptions that can be made are an in this review it has been watched that the number of info yield designs exhibited in every emphasis has a critical impact on the preparation and test information correctness. To study this impact we have utilized the taking after equation: NS $=$ NPR $\times$ NTSP Where, Ns = add up to number of info yield designs NPIt $=$ number of introductions NTS $p=$ number of info yield models per introduction (9) The aftereffects of Table 1 shows that for a blend of NPR=1 and NTSP=30 the \%MAE is least. In this way, it is comprehended that for the present review BATCH Handling is thought to be best. TABLE 1: input=Mean and SD, output FMax, NIT450, q 4.4, a 4.7, No. of Hidden layer hubs $=11$ 


\begin{tabular}{|c|c|c|c|}
\hline NPR & NTSP & RMSE & MAE\% \\
\hline 30 & 1 & 0.0725 & 71.48 \\
\hline 15 & 2 & 0.1318 & 29.06 \\
\hline 6 & 5 & 0.1335 & 33.45 \\
\hline 5 & 6 & 0.1301 & 44.9 \\
\hline 2 & 15 & 0.1722 & 23.38 \\
\hline I & 30 & 0.1836 & 11.15 \\
\hline
\end{tabular}

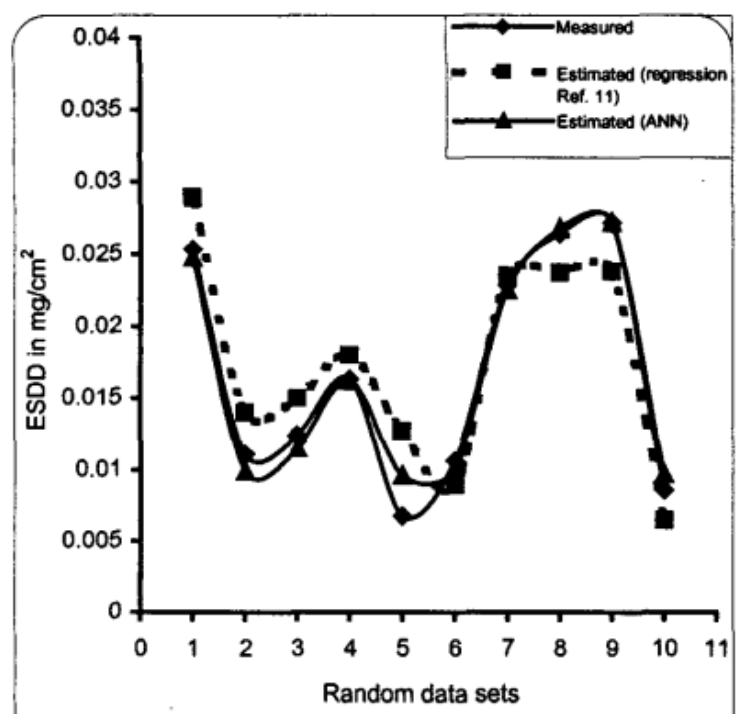

Figure 2. Comparison of reference measured and estimated values of salt contamination

\section{Conclusion}

In this paper, ANN has been connected effectively in disease seriousness estimation studies for capacity estimation, which is a noteworthy ANN application. Displaying of the compound non-direct capacity \&LID = f fl RJ PI WV), the condition which is obscure, has been proficient precisely. Assist relative investigation of the assessed comes about with the deliberate information gathered from the site estimation plentifully illustrate the viability of the utilisation of ANN in displaying a framework from the consequences of the location estimate as it were, where this present reality structure has a complicated nonlinear relationship. The estimation of primary sullying level as far as ESDD will help in the foundation of upkeep strategy and for tending to a viable arrangement against contamination flashover of high voltage separators.

\section{References}

[1] K Haribabu, GRKS Subrahmanyam, D Mishra. A robust digital image watermarking technique using auto encoder based convolutional neural networks. IEEE Workshop on Computational Intelligence: Theories, Applications and Future Directions (WCI). Kanpur. 2015; 1-6.

[2] T Ren, S Liu, G Yan, H Mu. Temperature prediction of the molten salt collector tube using BP neural network. in IET Renewable Power Generation. 2016; 10(2): 212-220.

[3] X Dong, T Tao, S Liu, Y Xia, Y Yu. Prediction of salt water intrusion using BP-RAGA coupled neural network model. 2010 3rd International Congress on Image and Signal Processing. Yantai. 2010: 4244-4248.

[4] AN Jahromi, AH El-Hag, EA Cherney, SH Jayaram, M Sanaye-Pasand, H Mohseni. Prediction of leakage current of composite insulators in salt fog test using neural network, CEIDP '05. Annual Report Conference on Electrical Insulation and Dielectric Phenomena. 2005; 309-312.

[5] ME Valle. A Robust Subspace Projection Autoassociative Memory Based on the M-Estimation Method, in IEEE Transactions on Neural Networks and Learning Systems. 2014; 25(7): 1372-1377. 
[6] PK Sethy, L Panda, SK Behera. Ann based image restoration in approach of multilayer perceptron. International Conference on Inventive Computation Technologies (ICICT), Coimbatore. 2016: 1-4.

[7] K Tamersit, F Djeffal. Modeling of a new graphene-based smart sensor for high performance $\mathrm{pH}$ monitoring applications. 8th International Conference on Modelling, Identification and Control (ICMIC), Algiers. 2016: 16-21.

[8] X B Huang, C Xie , H Li. Equivalent Salt Deposit Density Optical Fiber Sensor for Transmission Lines in Power Grid, in IEEE Sensors Journal. 2017; 17(1): $91-99$

[9] Vinothini S, Hussain AJ, Jayaprakashvel. M Bioprospecting of Halotolerant Marine Bacteria from the Kelambakkam and Marakkanam Salterns, India for Wastewater Treatment of Plant Growth Promotion. Biosciences Biotechnology Research Asia. 2014; 11, 313-321.

[10] Muthezhilan R, Ragul R, Pushpam AC, Hussain AJ. Production and Purification of Biosurfactant from Marine Yeast Isolated from Kelambakkam Salterns. 2014.

[11] Gibet Tani Hicham, El Amrani Chaker, Elaachak Lotfi. Comparative Study of Neural Networks Algorithms for Cloud Computing CPU Scheduling. International Journal Of Electrical And Computer Science. 2017.

[12] Wayan Oger Vihikan,Ketut Gede Darma Putra, Putu Arya Dharmaadi. Foreign Tourist Arrivals Forecasting Using Recurrent Neural Network Backpropagation Through Time. TELKOMNIKA Telecommunication, Computing, Electronics and Control. 2017. 